\title{
Chemical hardness and density functional theory
}

\author{
RALPH G PEARSON \\ Chemistry Department, University of California, Santa Barbara, CA 93106, USA
}

\begin{abstract}
The concept of chemical hardness is reviewed from a personal point of view.
Keywords. Hardness; softness; hard \& soft acids bases (HSAB); principle of maximum hardness (PMH) density functional theory (DFT).
\end{abstract}

\section{The HSAB principle}

The original basis for the concept of chemical hardness lies in some observations by inorganic chemists. Some metal ions form very stable complexes in aqueous solution with iodide ions, and weak, or no complexes, with fluoride ions. Examples would be $\mathrm{Hg}^{2+}$ and $\mathrm{Pt}^{2+}$. Other metal ions such as $\mathrm{Mg}^{2+}$ or $\mathrm{Al}^{3+}$ form stable complexes with $\mathrm{F}^{-}$, but not $\mathrm{I}^{-}$, in water. Also, $\mathrm{Mg}^{2+}$ and $\mathrm{Al}^{3+}$ occur in nature as oxide or carbonate ores, where $\mathrm{Hg}^{2+}$ and $\mathrm{Pt}^{2+}$ occur as sulfide or arsenide ores.

In 1962, John Edwards and I published a paper on nucleophilic reactivity. We pointed out that some substrates react rapidly with strongly basic nucleophiles, such as $\mathrm{OH}^{-}$, but not with polarizable nucleophiles, such as $\mathrm{I}^{-}$or thiourea. Examples would be acyl halides or phosphate esters. Other substrates, such as hydrogen peroxide or Pt (II) complexes, react rapidly with $\mathrm{I}^{-}$or thiourea, but very slowly with $\mathrm{OH}^{-}$, the strongest base possible in water.

At this time (the 1960's) it was very popular to look at chemistry in terms of the generalized acid-base concept of G N Lewis,

$$
\mathrm{A}+: \mathrm{B} \leftrightarrow \mathrm{A}: \mathrm{B} .
$$

The Lewis acid, A, is an electron acceptor or electrophile. The base :B is an electron donor (the two dot or a nucleophile). Almost all inorganic compounds, such as $\mathrm{CuCl}_{2}$ or $\mathrm{CuCl}_{4}^{2-}$, can be considered acid-base complexes, A:B. This is also true of organic compounds, such as $\mathrm{CH}_{3} \mathrm{Br}$ or $\mathrm{CH}_{3} \mathrm{COOH}$. Thus, (1) covers the entire range of chemistry. Any general statement that can be made about the strength of the chemical bond in A: B will be of great use. While bond energies are thermodynamic data, kinetic or rate data can also be included by considering the activated complex as an example of A : B. The effect of a strong coordinate bond is to lower the activation energy.

With this background, I looked at a large number of Lewis acids to see if they behaved like $\mathrm{Mg}^{2+}$, or like $\mathrm{Hg}^{2+}$. Alternatively, whether they behave like $\mathrm{CH}_{3} \mathrm{COCl}$ or $\mathrm{H}_{2} \mathrm{O}_{2}$, in terms of rate data. A similar thing was done for the common bases; for example, do they bind better to $\mathrm{Hg}^{2+}$ or to $\mathrm{Mg}^{2+}$.

The important factor for bases is the nature of the donor atom. Putting them in the order of increasing electronegativity gives

$$
\text { As }<\mathrm{P}<\mathrm{Se}<\mathrm{S} \sim \mathrm{I} \sim \mathrm{C}<\mathrm{Br}<\mathrm{Cl}<\mathrm{N}<\mathrm{O}<\mathrm{F} .
$$

The donor atoms to the left are the ones that bonded well to $\mathrm{Hg}^{2+}$ and $\mathrm{Pt}^{2+}$, and those to the right bond well to $\mathrm{Mg}^{2+}$ and $\mathrm{Al}^{3+}$.

A large number of Lewis acid were put into one of two boxes, eventually labeled hard and soft. The same was done for a number of common bases. The classification was done empirically, but because of a shortage of data, all of one kind, a variety of criteria were used. These included bond energies, equilibrium constants, rates of reaction and even the existence or non-existence of certain compounds.

The properties of the acids and bases in the various boxes were such that it seemed reasonable to label the boxes as hard or soft. In general terms we had the following definitions: (1) soft acid - the acceptor atom is of low positive charge, large size and has polarizable outer electrons; (2) hard acid - the acceptor atom is of high positive charge, small size and has no easily polarized outer electrons; (3) soft base - the donor atom is of low electronegativity, easily oxidized, highly polarizable and with low-lying empty orbitals; (4) hard base - the donor atom is of high electronegativity, hard 
to oxidize, of low polarizability and with only high energy empty orbitals.

Such qualitative description did not allow for the quantification of hardness or softness. Acids and bases were put into one of two categories, but there was no way to rank order within the boxes. It was still possible to summarize a great deal of diverse chemical information by the principle of hard and soft acids and bases (HSAB): hard acids prefer to coordinate to hard bases and soft acids prefer to coordinate to soft bases.

The HSAB principle is an empirical one, which simply restates the very information that caused each acid or base to be placed in its proper box. It depends on no theory for its validity. Nevertheless, it was very clear that soft-soft combinations depended mainly on covalent bonding and hard-hard combinations mainly on ionic bonding.

The inability to define hardness in an exact way, and to assign numerical values to it was a very serious drawback and led to much well-deserved criticism. Many empirical scales were proposed, but none were of general utility, or with a sound theoretical base.

A complicating feature was that there is at least one other property of an acid or a base that determines its behavior. For want of a better term, this may be called the intrinsic strength. Thus $\mathrm{H}_{2} \mathrm{O}$ and $\mathrm{OH}^{-}$are both hard bases, but the latter forms stronger bonds to virtually all acids than the former. Also $\mathrm{Mg}^{2+}$ and $\mathrm{Na}^{+}$ are both hard acids, but $\mathrm{Mg}^{2+}$ is the stronger acid. The strength of the coordinate bond in $\mathrm{A}$ : B depends primarily on the intrinsic strengths of $\mathrm{A}$ and $\mathrm{B}$. These, in turn, depend mainly on charge and size considerations. The HSAB Principle is really stating that there is an extra stabilization in a hard-hard combination, or a soft-soft one. In practical terms, it is predicting that for an exchange reaction, which is what is observed in the laboratory,

$$
A: B^{\prime}+A^{\prime}: B \leftrightarrow A: B+A^{\prime}: B^{\prime} .
$$

$\Delta H$ will be negative, if the partners are matched:

$$
h s+s h=h h+s s, \quad \Delta H<0 .
$$

Pauling had proposed bond energy equations, which could be used to predict $\Delta H$ for reaction (2). I noticed that his equation almost always failed. It not only missed the value of $\Delta H$ badly, even the sign was wrong! Since the Pauling bond energy equation was not only famous, but was still being used, I wrote an article about its failure. It created a stir in the public press for a few days. The New York Times had column headlines, which said "Pauling's Equation Found to be Useless". The reaction among chemists was almost zero. Very few chemists believed that Pauling could do anything wrong.

Actually, Pauling had used his equation to assign electronegativity values to the elements. He got these right, even with a poor equation. This was a tribute to his great chemical insight.

The HSAB Principle is very good at predicting the sign of $\Delta H$ correctly, but not its magnitude. It is not infallible, however, if we try to compare acids or bases of quite different intrinsic strengths. Steric factors and solvation energies can also be complicating effects.

In spite of these problems, the HSAB concept has proved to be very useful in chemistry and related fields. Two of the 25 papers my coworkers and I wrote on the subject have been listed as among the 125 most cited in the 125-year history of the African Chemical Society. The original article and one other have also been identified in current contents as citation classics. For many chemists the words hard and soft seemed to fill a need in their chemical vocabularies.

The reason for the existence of HSAB was also fairly well understood. Hard acids and bases are well suited for strong ionic bonding. Soft acids and bases are better suited for covalent bonding, including $\pi$-bonding. Obviously it is better to have the pairing match up the bonding preferences.

\section{The advent of density functional theory}

The really serious objection to the HSAB concept was that no exact definition of hardness existed. Nor was there an operational definition linking hardness to experiment. As a result, there was no way to give a theoretical or experimental value to the hardness for any chemical system. A chemical system is any collection of nuclei and electrons. For many chemists, quantities, which could not be measured and numbers assigned, had no place in exact science. This view is not completely valid, since all sciences use some terms that are not quantitative. An example would be "solvent polarity", which is hard to define, though a very useful description.

Measuring the electronic absorption spectrum of a substance in also useful, and can be very quantitative. But surely there is some information if you say that a substance is red! In fact, the idea of the hardness in the old days was an example of fuzzy logic. This 
is a respectable branch of mathematics in which you have some information, but not enough for a definite conclusion.

Still it was a great step forward when Bob Parr finally explained to me what I meant by chemical hardness. He and his colleagues had already (1978) published a pioneering paper on the application of DFT to chemical systems.

What Parr showed was that for every chemical system there is a quantity $\mu$, called the electronic chemical potential. The chemical potential must be constant everywhere in such a system. It also has the important and defining property given by

$$
\mu=(\partial E / \partial N)_{v}=(\delta E / \delta \rho)_{v},
$$

where $E$ is the electronic energy, $N$ is the number of electrons and $v$ is the potential due to the nuclei. The symbolism of the second part of (4) refers to the functional relationship of $E$ to the electron density $\rho$.

To understand (4), figure 1 shows a plot of $E$ vs $N$ for any arbitrary system. The energies are all negative with zero energy high up on top. We only know the energy experimentally for integral values of $N$ from data such as ionization potentials $I$ and electron affinities $A$. However, it is convenient to think of a smooth curve connecting the known values. In a molecule it is common to think of each atom, for example, having a non-integral number of electrons.

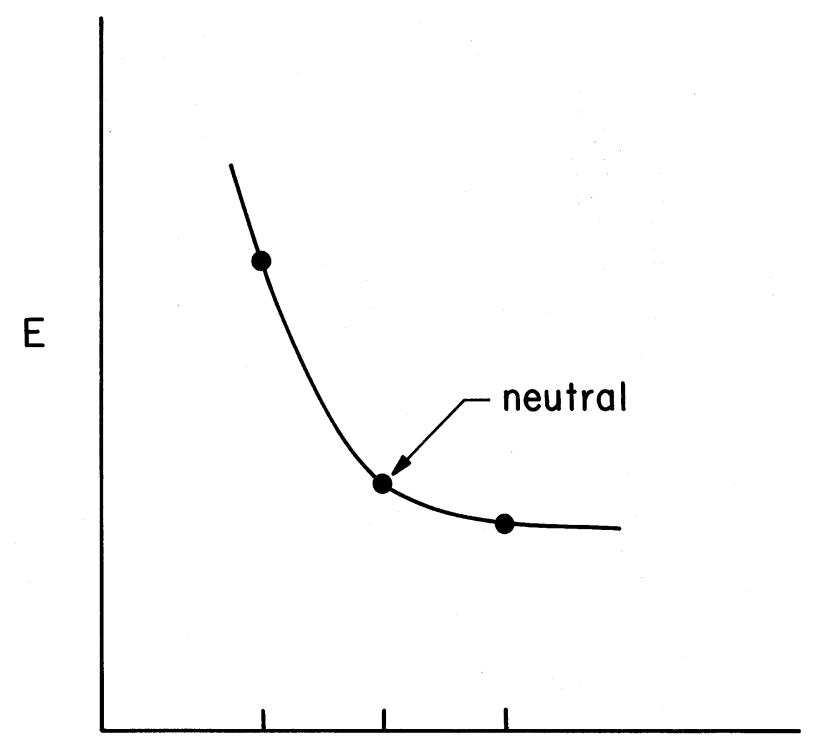

$\mathrm{N}$

Figure 1. Plot of the total electronic energy vs the number of electrons for a fixed collection of nuclei.
Now $\mu$ is simply the instantaneous value of the slope of figure 1 at any value of $N$. If we pick the neutral species (or any other) as our starting point, the method of finite differences can be used to estimate the slope

$$
-\mu=-(\partial E / \partial N)_{v} \approx(I+A) / 2=\chi_{M} .
$$

$(I+A) / 2$ is simply the Mulliken electronegativity $\chi_{M}$. Because of the fundamental relationship to the chemical potential, $\chi_{M}$ may be called the absolute electronegativity. It is important to realize that $\chi_{M}$, or just $\chi$, is now quite different from Pauling's original meaning of electronegativity, which was a property of an atom in a molecule. Now $\chi$ is a property of the entire molecule, atom, ion or radical. This alters the uses of $\chi$ dramatically.

Chemical hardness and DFT came together in 1983 when Parr spent a sabbatical quarter at the Institute for Theoretical Physics in Santa Barbara. He showed me figure 1 and asked if the curvature might have something to do with hardness. The curvature in the way in which the slope changes with $N$. From the method of finite differences, the curvature in equal to $(I-A)$. Since $I$ and $A$ are known for most atoms, $I$ was able to say, after a few quick calculations, that the curvature was exactly what I meant by hardness!

Fortunately the Greek letter $\eta$, or eta $=h$, was available, and could be used for hardness. The softness is simply the reciprocal of the hardness, and is called $\sigma$, the Greek letter for $s$.

Within a few days we wrote a paper, which rigorously defined $\eta$ and gave an empirical method of evaluating it:

$$
\eta=\frac{1}{2}\left(\frac{\partial \mu}{\partial N}\right)=\frac{1}{2}\left(\frac{\partial^{2} E}{\partial N^{2}}\right)=\frac{(I-A)}{2} .
$$

The factor of one-half was put in to symmetrize with $\chi$. As it turned out, it would have been better not to include it, and recent usage has dropped it from the definition. I will adopt this sensible change in what follows.

For two reactants, $\mathrm{A}$ and $\mathrm{B}$, we can write

$$
\begin{aligned}
& \mu_{A}=\mu_{A}^{0}+\eta_{\mathrm{A}} \Delta N, \\
& \mu_{B}^{0}=\mu_{B}^{0}+\eta_{B} \Delta N,
\end{aligned}
$$

where $\Delta N$ is the number of electrons transferred from the base to the acid. 
Applying the condition that $\mu_{A}=\mu_{B}$, we find.

$$
\Delta N=\frac{\left(\mu_{B}^{0}-\mu_{A}^{0}\right)}{\left(\eta_{A}+\eta_{B}\right)}=\frac{\left(\chi_{A}^{0}-\chi_{B}^{0}\right)}{\left(\eta_{A}+\eta_{B}\right)} .
$$

Clearly the acid must be more electronegative than the base. The difference in absolute electronegatives drives the electron transfer, and the sum of the hardness parameters acts as a drag or resistance.

This was very satisfactory, since the common meaning of hardness is resistance to change or deformation. Also, an explanation of the HSAB principle emerges. Two soft molecules would have a large value of $\Delta N$. The transferred electrons are used to form the coordinate covalent bond between $\mathrm{A}$ and B. Two hard molecules would have small $\Delta N$, little covalent bonding, and a reliance on ionic bonding instead.

In spite of this, it was by no means clear that (6) was a valid definition of the hardness implied by the HSAB concept. Only for cations, atoms and a few molecules and radicals could it be shown to give reasonable results. For anions, the approximation $\eta=$ $(I-A) / 2$ was clearly not valid. For molecules there were many values of $I$ available, but fewer values of $A$.

Most common molecules, in fact, have negative electron affinities. Parr and I had decided to use the adiabatic value, $A=0$, for these cases, since negative values of $A$ did not seem to be reliable. However, (6) stated clearly that it was the vertical values of $A$, the negative ones, that were required. Setting $A$ equal to zero did not give good results in most cases.

At this point there were some timely and fortunate developments. The technique of electron transmission spectroscopy had already been invented, to give accurate values of negative electron affinities. In fact, a number of results had already been published, though most chemists, including Parr and myself, were unaware of them. At the same time many new values of positive $A$ were being published. All the new data gave much better results for molecules and free radicals, when (6) was used.

For ions, both positive and negative, there are serious problems in using (6). Except for monatomic cations, the data are unavailable. You cannot measure $A$ for $\mathrm{Br}^{-}$, since $\mathrm{Br}^{2-}$ does not exist. In general, it is not possible to add or remove a second electron from a small molecule to form a stable species. Also, the finite difference method used to derive (6) is not appropriate. It weights the gain or loss of an electron equally. This is not the case for ions, where there will be a strong bias for an unidirectional flow of electrons.
Unlike the chemical potential, $\eta$ is not constrained to be constant everywhere. The hardness will have local values, and $\eta$ will simply be a global average. Parr and his coworkers defined the local hardness.

$$
\tilde{\eta}=\frac{1}{2}(\delta \mu / \delta \rho)_{v} .
$$

This measures the change in chemical potential with electron density in different parts of the molecule. The calculation of the local hardness is difficult. The local softness, is easier to work with:

$$
\tilde{\sigma}=(\delta \rho / \delta \mu)_{v}=\sigma f .
$$

Here $f$ is the frontier orbital density, called the Fukui function.

There are usually two frontier orbitals, depending on the direction of electron flow.

$$
\begin{aligned}
& f=\rho_{\text {номо }}, \quad \text { donor molecule } \\
& f=\rho_{\text {LUMO }}, \quad \text { acceptor molecule, } \\
& f=\frac{1}{2}\left(\rho_{\text {HОМО }}+\rho_{\text {LUMO }}\right) \text { both donor and acceptor. }
\end{aligned}
$$

The factor of one half is approximate. In general, $f$ will be some weighted combination of $\rho_{\text {номо }}$ and $\rho_{\text {LUMо }}$. Though $\tilde{\eta}$ and $\tilde{\sigma}$ are related, one is not simply the reciprocal of the other. There is one way of estimating the local hardness at each atom in a polyatomic system. This is based on the electron density distribution leading to a net charge on the atom. The local hardness increases with the net positive charge.

Returning to the reaction of $\mathrm{A}$ and $\mathrm{B}$, we can expand the energy of the combined system as a TaylorMacLaurin series in $\Delta N$, and find $\Delta E$ :

$$
\Delta E=\Delta \mu \Delta N+\left(\eta(\Delta N)^{2} / 2 .\right.
$$

Here $\Delta \mu=\left(\mu_{A}^{0}-\mu_{B}^{0}\right)$ and $\eta=\left(\eta_{A}+\eta_{B}\right)$. The largest decrease in the energy will be when $\Delta N$ is given by (6). The energy change is given by

$$
\Delta E=-(\Delta \mu)^{2} / 2 \eta=\Delta N \Delta \mu / 2 .
$$

If this energy change is calculated, it will be only a small fraction of the total energy change due to forming a bond, A : B. The reason is that it only gives the initial effects of bringing $A$ and $B$ together, there will be large charge density changes and energy effects due to 
ionic and covalent bonding. These are not included in (14). For the same reason the calculated value of $\Delta N$ cannot be used to find the final charges on $\mathrm{A}$ and $\mathrm{B}$.

In spite of these shortcomings, $\Delta N$ has proved very useful in correlating the rates of reaction between a series of similar reactants with a common substrate. A large value of $\Delta N$ is a harbinger of a strong interaction. It indicates a lowering of the activation energy, and in some instances, the formation of a strong chemical bond.

\section{Correlation with molecular orbital theory}

The most widely used theory by chemists is the molecular orbital (MO) theory. It is important that $\eta$ and $\mu$ be put into a MO framework. This can readily be done within the limitations of Koopmans' theorem, the orbital energies of the frontier orbitals are given by

$$
-\varepsilon_{\mathrm{HOMO}}=I, \quad-\varepsilon_{\mathrm{LUMO}}=A .
$$

Figure 2 shows an orbital energy diagram for $\mathrm{Br}_{2}$, $\mathrm{NH}_{3}$ and atomic Fe. Only the HOMO and LUMO orbitals are shown. These are called the frontier orbitals. The absolute $E N$ is shown as a dashed line halfway between the HOMO and the LUMO. The hardness is the energy gap between the two frontier orbitals. We can see that $\mathrm{Br}_{2}$ is more electronegative than either

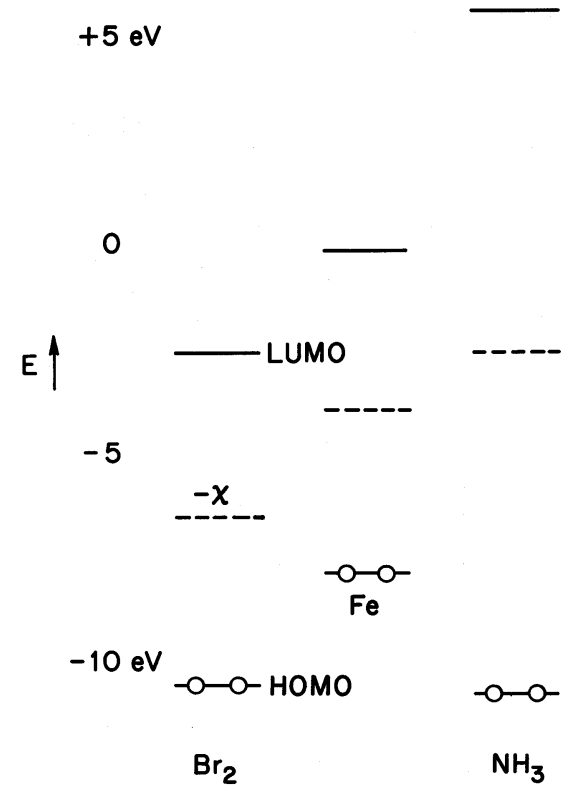

Figure 2. Molecular orbital energy diagram for $\mathrm{Br}_{2}$, atomic $\mathrm{Fe}$ and $\mathrm{NH}_{3}$, showing the HOMO, LUMO and $\chi$. The hardness in each case is the HOMO-LUMO gap.
$\mathrm{Fe}$, or $\mathrm{NH}_{3}$, and will accept electrons from both of them. Also $\mathrm{NH}_{3}$ is much harder than $\mathrm{Fe}$ or $\mathrm{Br}_{2}$. This is a result of large negative electron affinity.

Figure 2 offers a concise and graphic definition of chemical hardness. A hard molecule has a large HOMO-LUMO gap and a soft molecule has a small HOMO-LUMO gap. This statement is, of course, consistent with the definition of (5) and (6). But it also agrees with the earlier, imprecise definitions.

In quantum theory, changes in the electron density of a chemical system result from the mixing of suitable excited-state wave functions with the groundstate wave function. The mixing coefficient is inversely proportional to the excitation energy between the ground and the excited state. A small HOMO-LUMO gap automatically means small excitation energies to the manifold of excited states. Therefore, soft molecules, with a small gap, will have their electron density changed more easily than a hard molecule. But this is the essence of the old definition.

In terms of chemical reactivity, we can conclude that soft molecules will be more reactive than hard molecules for unimolecular reactions such as isomerization and dissociation. For bimolecular reactions, it is convenient to use reaction (1) as the model. From perturbation theory, we know there are three main bonding interactions in (1). They are (a) electron delocalization, (b) polarization, and (c) classical electrostatic. The first is greatest when $I$ is small for both reactants, and $A$ is large and positive. But this means both molecules should be soft. The same is true for polarization, which is a mutual effect.

Hard reactants, where the electron density is hard to change, must rely on classical electrostatic effects, or ionic bonding. We have an explanation for the HSAB principle, which is consistent with older explanations.

But we are also reminded that donated electrons must come from a definite filled orbital and go into a definite empty orbital. Also, (12) tells us that in a MO, different atoms have different local softness, and different tendencies to react. The net charges on the atoms will determine the reactive sites for ionic bonding.

\section{The principle of maximum hardness}

The foregoing certainly suggests that hardness is a good thing for stability in any chemical system. There is a large amount of evidence of various kinds, which supports this conclusion. In 1987 I said "There seems to be a rule of nature that molecules arrange 
themselves to be as hard as possible". This can be taken as a condensed statement of the "Principle of Maximum Hardness", or PMH.

The kind evidence that I had in mind at the time was twofold: a common rule was that maximum stability ion a chemical system was achieved when all the bonding MOs were filled, and all the anti-bonding MOs were empty; quantum mechanical molecular energy calculations always found the most stable structure had the largest energy gap between the HOMO and the LUMO.

More qualitative evidence can be found with a little effort. For example, the hardness always increased when strong bonding occurred between reactive partners. A and B. When the energy is falling as the distance between A and B decrease, the HOMO is going down in energy, and the LUMO is going up in energy. But when the energy is rising along a reaction coordinate, the HOMO is going up in energy (due to filled orbitals interacting), and the LUMO is little affected.

In LCAO-MO theory, it can be proven that the best values of the mixing coefficients will be those that minimize the HOMO energy and maximize the LUMO energy. This holds for simple cases, like Huckel theory, but also for Hartree-Fock calculations, provided they obey the SCF constraints.

Parr and Chattaraj have given a general proof of the PMH. They also showed a serious restriction, in that the values of $\mu$ and $v$ (the nuclear potential) should remain constant. A number of molecular energy calculations have shown that the PMH is indeed valid, if these conditions are met. However, it also remains true in many cases where the restrictions are not obeyed. Failure to understand this has hindered our attempts to use hardness, instead of energy, as a stability criterion.

The transition state (TS) theory of chemical reactions leads to some interesting conclusions. Clearly, the TS is a very unstable chemical system, since it decomposes immediately. Therefore, it must be very soft, and have a small HOMO-LUMO gap. There is a Principle of Maximum Softness for transition states. This has been confirmed in a number of cases, but exceptions can occur.

Excited states are always softer than ground states. The excitation energy can be electronic, vibrational, rotational or translsational. Any energy above the ground state energy makes it easier to distort the electron cloud. This agrees with the $\mathrm{PMH}$, since it means that the equilibrium state is harder than any non-equilibrium state. The proof is based on the as- sumptions that the same ions are reached, whether we start with the ground state system or the excited state. The chemical potential is unchanged, since $I$ is decreased and $A$ is increased, but the hardness is decreased by twice the excitation energy. This agrees with the PMH, the equilibrium state is harder than the non-equilibrium state. We even see that $\mu$ must remain constant. Since excited states have different bond distances from ground states and may even have different structures, $v$ is not necessarily constant.

\section{Closed systems}

A closed chemical system is one, which does not exchange matter or energy with its environment. In the preceding, we have implicitly been dealing with open systems, since the electron affinity requires the adding of one more electron to the system. What changes will occur, if $A$ is no longer allowed to play a role? The question really relates back to figure 1 , since it is no longer valid to use finite differences to define the slope and curvature.

Continuing to use (5) to define the chemical potential, we conclude that in a closed system

$$
\mu=-I=\varepsilon_{\text {номо }} .
$$

Note that Koopmans' theorem works better than expected because the constraint that $v$ is constant means that the vertical ionization potential is used. There is still an error due to the relaxation of the electron cloud after ionization, however.

The orbital energy can be written as

$$
\varepsilon=v_{n}+v_{e}+t=\mu,
$$

where $v$ is written as $v_{n}$ to emphasize that it is the nuclear potential, which is negative. The next two terms are positive. They are the total potential due to the other electrons, and the local kinetic energy of one electron in the particular orbital involved.

If we had the exact electron density for the system, we would have the exact orbital energy and chemical potential, which would be constant everywhere. For an approximate density, $\mu$ would not be constant everywhere. Electron density would move from regions where $\mu$ was too positive to regions where $\mu$ was too negative, until equilibrium was reached and the energy minimized.

What about the hardness of a closed system? Looking at figure 1 , we see that the slope is always 
negative, as must $\mu$ be, and the curvature is always positive as $\eta$ must be. Table 1 clarifies this by giving the experimental values of the energy as a function of $v$ for the carbon atom. Adding the first electron to a bare nucleus gives a large drop in energy. But adding more electrons produces smaller and smaller decreases. Clearly, each added electron shields the nucleus somewhat and reduces its effect.

This means that $\mathrm{v}_{e}$ in (17) is part of the hardness, since it cancels part of $v_{n}$. But the kinetic energy also plays a role. If we invoke the virial theorem, we find for changes in energy,

$$
\Delta E=\Delta V / 2=-\Delta T,
$$

so the kinetic energy must increase. This agrees with equations (13) and (14), where the first term is negative, and the second, positive term is the change in kinetic energy.

It is $v_{n}$ which creates a negative value for $\mu$. But it cannot be part of the hardness, since it is the potential of the nuclei, which are fixed in position. The effect on the chemical potential is independent of changes in $\rho$. It is tempting, then, to assume that $v_{e}$ and $t$, which do depend on $\rho$, fix the hardness $\eta$. For the average, or global hardness, we will expect than $\eta$ is some simple function of $\left\langle v_{e}\right\rangle$ and $\langle t\rangle$.

To find the function we will try to improve the energy of an approximate wave function. An example is the simple hydrogenic wave function for the ground state helium atom, with effective nuclear charge $\alpha=1.6875$. The local orbital energy is given by

$$
\mu=\varepsilon=\frac{2}{r}+\frac{1}{r}-\frac{e^{-2 \alpha r}}{r}(1+\alpha r)-\frac{\alpha^{2}}{2}+\frac{\alpha}{r} .
$$

As expected, $\mu$ or $\varepsilon$ is not constant. Its average value is - 0.8965 a.u.

Equation (19) gives $\varepsilon=-0.8965$ at $r=0.31$ and $r=1.41$ a.u. It is more negative than $\langle\varepsilon\rangle$ at $r$ less

Table 1. Electronic energy of the carbon atom as a function of the number of electrons.

\begin{tabular}{cccc}
\hline $\mathrm{N}$ & $-E(\mathrm{eV})$ & $N$ & $-E(\mathrm{eV})$ \\
\hline 0 & 0 & 4 & $994 \cdot 3$ \\
1 & $489 \cdot 9$ & 5 & $1018 \cdot 7$ \\
2 & $882 \cdot 0$ & 6 & $1030 \cdot 0$ \\
3 & $946 \cdot 5$ & 7 & 1031.3 \\
\hline
\end{tabular}

than 0.31 and greater than 1.41 . We can lower the energy, according to (14), by moving electron density from the region between these two limits, to the inner and outer regions. The energy lowering is

$$
\Delta E=-\frac{\left\langle(\varepsilon-\langle\varepsilon\rangle)^{2}\right\rangle}{2 \eta}=\frac{\delta^{2}}{2 \eta} .
$$

It is convenient to use the variance, $\delta$, which is $\left(\left\langle E^{2}\right\rangle-\langle E\rangle^{2}\right)^{1 / 2}$. This has been used in the past as a measure of goodness for trial wave functions. It is zero for the exact density function.

Since the energy of the single $-\zeta$ function is -2.8477 a.u., and the exact energy of $\mathrm{He}$ is -2.9037 , $\Delta E$ can be anything less than $-0 \cdot 0560$. The sum of $\left\langle v_{e}\right\rangle$ and $\langle t\rangle$ is 2.479 a.u. for He. If it is assumed that

$$
\eta=2\left\langle v_{e}+t\right\rangle
$$

then the corrected energy becomes $-2 \cdot 8625$, which is very close to the Hartree-Fock value $(-2 \cdot 8617)$. This is probably the best that can be done with (20).

The reason is that the remaining correction is the correlation energy. To find this requires a correlated wave function, which is not the case with our simple example. We can easily check this deduction by taking the case of He with a double $-\zeta$ function. This gives essentially the HF energy without correction. Using (20) we find that $\delta^{2}=0$, to four decimal places. As expected it cannot be corrected further. If we use (20) on a correlated wave function, we get a large value of $\delta^{2}$, and an overcorrected energy.

Equation (21) has been tested in a number of cases, and found to give quite good results, if we remember the HF limitations. Still it is essentially semi-empirical. It would be god to find a rigorous derivation of it. This has not yet been done.

It must also be recognized that (14), on which (20) rests, is not exact. It requires that $\Delta N$ be small, for one thing, and it uses the global average hardness, instead of the local value, for another. Fortunately, we can test (14) in another way, to see where it might fail. We can use the alternative equation for the energy lowering,

$$
\Delta E=\Delta N \Delta \mu / 2 .
$$

In (22) $\Delta \mu$ has the same meaning as before. But $\Delta N$ is calculated by introducing an improved wave function, which gives an improved electron density, $\rho_{c}$, compared to our original density, $\rho$, for the single $-\zeta$ 
function. For each volume element, the product $\rho \mathrm{d} V$ gives the small fraction of an electron $\mathrm{d} N$, contained. We must integrate over all space to get a finite value. Comparing $\rho \mathrm{d} V$ with $\rho_{c} \mathrm{~d} V$ given the change in $\mathrm{d} N$. Integration gives $\Delta N=0$, since both $\rho$ and $\rho_{c}$ are normalized to $N$.

However, if we weight each volume element by the local chemical potential, $\mu$, we get a non-zero result. If $\left(\rho_{c}-\rho\right)$ is negative then $d N$ is negative and equals the fraction of an electron with chemical potential $\mu$ that disappears. When $\left(\rho_{c}-\rho\right)$ is positive, $d N$ is the fraction of an electron that has appeared at a different chemical potential. If the improved wave function has been picked wisely, then the difference in chemical potential will be negative, and the energy will decrease.

The chemical potential is that given by (19), and is for the single $-\zeta$ function. The working equation is

$$
2 \Delta E=\langle\Delta N \Delta \mu\rangle=\int \rho_{c} \varepsilon \mathrm{d} V-\langle\varepsilon\rangle .
$$

Remarkably, we have found the energy of the improved wave function using only its associated density function and the local orbital energy of a simpler function, whose energy we know.

As an example of the improved function, we take the two $-\zeta \mathrm{HF}$ function. Using (23) we calculate $\Delta E=-0.0143$ a.u. Adding this to $-2 \cdot 8477$, we find $E=-2.8620$, which is very close to the Hartree-Fock value. If we choose a correlated wave function, however, we find that the calculated $\Delta E$ is much less than needed. We can only correct to the HartreeFock level at best.

In spite of this limitation, (23) has potential utility. Like the virial theorem, it is an additional condition that must be satisfied. It can be used to help find improved variations of a simple trial function. Only the improved density function is needed.

\section{Conclusion}

We have tried to show that chemical hardness is a useful concept for understanding the behaviour of chemical systems. It is coupled with the electronic chemical potential, or absolute electronegativity, in this respect. In fact, it was at one time called the absolute hardness, to emphasize the connection. The term "absolute" is appropriate for the electronegativity, since there are many scales of EN, but only this one with a firm foundation in theory.

However, the use of "absolute" in describing the hardness is less justified. The only other scales of hardness are those measuring resistance to mechanical deformation, or physical hardness. Hence the name "chemical" hardness is very appropriate. Essentially, it measures the resistance to change in the electron distribution in a collection of nuclei and electrons. The definition of hardness by (6), was a major step in the history of chemical hardness. It brought the ill-defined hardness of HSAB into the regime of density functional theory. It also gave an operational definition of $\eta$ as $(I-A)$. The identification of $\eta$ with the HOMO-LUMO gap of molecular orbital theory has been richly rewarding in terms of understanding. In solids, this is the band gap, suggesting new approaches in solid-state theory.

Using MO theory again, we can write

$$
\eta=\left(\varepsilon_{\text {LUMO }}-\varepsilon_{\text {HOMO }}\right) .
$$

This is useful, since it is usually much easier to calculate the orbital energies of a molecule, than to measure its ionization potential and electron affinity. Great care must be taken in using (24), however. While the orbital energies of occupied orbitals can be calculated well, the energies of the so-called virtual orbitals are not.

Very stable molecules, such as $\mathrm{H}_{2} \mathrm{O}$ and $\mathrm{CH}_{4}$, have negative values of $A$, which usually cannot be calculated with any accuracy. For positive values of $A$, the deficiencies of Koopmans' theorem can be avoided by calculating the orbital energies of the system with one extra electron. Also the virtual orbital energies can sometime be useful in that the relative values for a series of related molecules will be put in the right order.

Certainly closed systems should be studied in more detail. Relating $\eta$ to the sum of $\left\langle v_{e}\right\rangle$ and $\langle t\rangle$, and to the HOMO-LUMO gap, as in (23) and (26), both have validity. Therefore there is a relationship between the two measures. The change in the electron density function is of paramount interest as chemical change occurs. The change in $\rho$ when an approximate wave function is improved, is also of great importance.

It appears that chemical hardness will be a subject of interest to the theoreticians for some time to come. This will certainly be true for the absolute electronegativity, or electronic chemical potential. We have DFT to thank for these useful innovations.

I have avoided giving references and mentioning names in this review. So many people have made valuable contributions to the subject of hardness, that it is difficult to choose among them. An exception is Bob Parr, since he is one of the fathers of chemical 
hardness. I would also like to mention my colleague and coauthor, William Palke. Bill passed away several years ago, a great loss to me and others.

There are two books that I need to mention. ${ }^{1,2}$ They were written with chemical hardness in mind. They contain the needed references, and also have much more in the way of rigorous theory and practical application.

Finally, I wish to thank Professor P K Chattaraj. His invitation gave me the chance to write this per- sonal account of a subject which has occupied much of my time and energy.

\section{References}

1. Parr R G and Yang W 1982 Density functional theory for atoms and molecules (New York: Oxford University Press)

2. Pearson R G 1997 Chemical hardness (Weinheim: John Wiley-VCH) 